\title{
Obstructive sleep apnea in children with Down syndrome: is it possible to predict severe apnea?
}

\author{
Mina Hizal ${ }^{1}$ (1) $\cdot$ Ozlem Satırer $^{2} \cdot$ Sanem Eryilmaz Polat ${ }^{1} \cdot$ Dilber Ademhan Tural $^{1} \cdot$ Beste Ozsezen $^{1} \cdot$ Birce Sunman $^{1}$. \\ Sevilay Karahan ${ }^{3} \cdot$ Nagehan Emiralioglu $^{1} \cdot$ Pelin Ozlem Simsek-Kiper ${ }^{4} \cdot$ Gulen Eda Utine $^{4} \cdot$ Koray Boduroglu $^{4}$. \\ Ebru Yalcin ${ }^{1} \cdot$ Deniz Dogru $^{1} \cdot$ Nural Kiper $^{1} \cdot$ Ugur Ozcelik $^{1}$
}

Received: 7 July 2021 / Revised: 9 September 2021 / Accepted: 12 September 2021 / Published online: 25 September 2021

(c) The Author(s), under exclusive licence to Springer-Verlag GmbH Germany, part of Springer Nature 2021

\begin{abstract}
The objectives are to explore the demographic and polysomnographic features of children with Down syndrome and to determine the predictive factors associated with severe sleep apnea. A total of 81 children with Down syndrome referred for full-night polysomnography were analyzed. In addition, parental interviews were performed for each child. Data were available for 81 children, with a mean age of 4.8 years. Severe obstructive sleep apnea was determined in 53.1\%. Age, sex, exposure to second-hand smoke, clinical findings, anthropometric features, and the presence of comorbidities were not predictors of severe obstructive sleep apnea. Children who were exposed to second-hand smoke had more sleep-related symptoms. Even in children without symptoms, the prevalence of severe obstructive sleep apnea was $40 \%$. Moreover, $86 \%$ of parents had no previous information regarding possible sleep breathing disorders in their children. Clinically significant central apnea was present in 10 patients $(12.3 \%)$.

Conclusion: Our results demonstrate that severe obstructive sleep apnea is common in children with Down syndrome, even in children without a history of symptoms of sleep apnea. It is not possible to predict patients with severe apnea; thus, screening of children with Down syndrome beginning from young ages is very important. Central apneas could be a part of the spectrum of sleep abnormalities in Down syndrome.
\end{abstract}

Keywords Down syndrome $\cdot$ Obstructive sleep apnea $\cdot$ Polysomnography $\cdot$ Sleep-disordered breathing $\cdot$ Central sleep apnea

\section{Abbreviations}

AHI Apnea-hypopnea index

BIPAP Bilevel positive airway pressure

BMI Body mass index

Communicated by Peter de Winter

Mina Hizal

minahizal@outlook.com

1 Department of Pediatric Pulmonology, Hacettepe University School of Medicine Ihsan Dogramaci Children's Hospital, Sihiye, Ankara, Turkey

2 Department of Pediatrics, Hacettepe University School of Medicine Ihsan Dogramacı Children's Hospital, Ankara, Turkey

3 Department of Biostatistic, Hacettepe University, Ankara, Turkey

4 Department of Pediatric Genetics, Hacettepe University School of Medicine Ihsan Dogramac1 Children's Hospital, Ankara, Turkey

$\begin{array}{ll}\text { CAI } & \text { Central apnea index } \\ \text { CHD } & \text { Congenital heart disease } \\ \text { CPAP } & \text { Continuous positive airway pressure } \\ \text { DS } & \text { Down syndrome } \\ \text { GI } & \text { Gastrointestinal } \\ \text { GERD } & \text { Gastroesophageal reflux } \\ \text { OSA } & \text { Obstructive sleep apnea } \\ \text { PSG } & \text { Polysomnography } \\ \text { SBD } & \text { Sleep breathing disorders } \\ \mathrm{SpO}_{2} & \text { Oxygen saturation values } \\ \mathrm{TST} & \text { Total sleep time }\end{array}$

\section{Introduction}

Down syndrome (DS), which is caused by an additional copy of chromosome 21 , is the most common chromosomal abnormality in children, occurring in an estimated one in 700 births [1-5]. Obstructive sleep apnea (OSA) is 
a frequent condition in people with DS, with an estimated rate between 55 and $97 \%$ [2, 6, 7]. There are many underlying predisposing factors for OSA in DS such as midfacial hypoplasia, relative macroglossia, glossoptosis, pharyngeal hypotonia, tonsillar hypertrophy, laryngomalacia, and obesity [8-10]. Additionally, many other comorbid conditions contribute to the occurrence of OSA in DS, including obesity, hypothyroidism, and gastroesophageal reflux (GERD) [11-13]. Untreated OSA has been associated with significant morbidities in children with DS, including IQ loss [14-17]. These have led to the recommendation of routine screening of these children by age 4 years, or sooner in symptomatic children [18-20]. However, it can be difficult to perform overnight polysomnography (PSG) in children with DS because it is often uncomfortable and poorly tolerated. [20-22].

It is not yet known which group of children with DS has a greater risk for severe sleep disturbance [6]. Parents of children with DS have been found to underestimate the presence of sleep abnormalities in their children [19]. Furthermore, alternative methods such as overnight pulse oximetry, questionnaires, and audio/video recordings have been reported to have limited use [23, 24]. Thus, few studies have attempted to identify factors associated with disease severity, and the results yielded were contradictory.[20, 25].

The aim of this study was to explore the demographic and PSG features of children with DS and to determine the predictive factors associated with moderate or severe sleep apnea. We hypothesized that we could predict patients with severe OSA either with demographic characteristics, clinical features, or comorbidities.

\section{Method}

\section{Participants}

Eighty-one children with DS (aged $<18$ years) who had full-overnight PSG performed at the Sleep Medicine Unit of Hacettepe University Children's Hospital from 2016 to 2020 were enrolled in the study. Children were referred based on symptoms of sleep disturbance and/or routine screening for DS, which was confirmed in all cases through genetic analyses. Both baseline and titration PSG results were reviewed. Patients with a total sleep time of fewer than $4 \mathrm{~h}$ or a technically inadequate PSG study were excluded. The study was approved by the ethics committee of Hacettepe University.

All patients in our unit were routinely evaluated by a respiratory physician before the PSG, and data were collected. Demographic data, PSG characteristics, sleep-related symptoms, comorbidities (congenital heart disease (CHD), hypothyroidism, feeding problems, anatomic functional and gastrointestinal (GI) conditions, asthma, allergic rhinitis, respiratory and airway problems), history of adenoidectomy and/or tonsillectomy, presence of subjective macroglossia on physical examination, use of supplemental oxygen or mechanical ventilation, and treatment information were collected retrospectively from the patients' medical records and also from records obtained previous PSG by respiratory physician of unit. In addition, during the data collection period of the study, family interviews were conducted by phone calls. The following information was questioned in the phone interviews: gestational week at birth, referral reason to the sleep unit, knowledge status about sleep disturbance, history of adenoidectomy and/or tonsillectomy, sleep-related symptoms at the time of PSG, presence of comorbidities, second-hand smoke exposure, and treatments. Anthropometric data were recorded before the PSG and body mass index (BMI), BMI centiles, and BMI-z-scores were calculated according to the age and sex of children aged over 2 years, and weight for age percentiles was calculated for children aged under 2 years based on DS-specific growth charts [26]. Patients were considered as obese if their percentile was $\geq 95$ th percentile for age and overweight if their percentile was 85th-95th for age [27]. Tonsillar hypertrophy was determined by records in accordance with the Brodsky categorization.[28].

\section{Polysomnography}

A standard overnight PSG (Alice 6 System, Philips Respironics Inc.) measurement included a 6-channel electroencephalogram $(\mathrm{F} 4, \mathrm{C} 4, \mathrm{O} 2, \mathrm{~F} 3, \mathrm{C} 3$, and $\mathrm{O} 1)$, bilateral electrooculograms (A1, A2), one submental and two tibial electromyograms, and a 6-lead electrocardiogram. Respiratory measurements included oxygen saturation using pulse oximetry, chest wall and abdominal movements using inductance pneumography, and airflow using a nasal cannula connected to a nasal pressure transducer. Polysomnograms were manually scored by a single certified experienced pediatric sleep medicine physician (U.Ö.) according to the criteria presented by the American Academy of Sleep Medicine.[29].

The apnea-hypopnea index (AHI) was defined as the number of apneas and hypopnea (obstructive, central, and mixed) per hour of total sleep time (TST). A diagnosis of OSA requires an $\mathrm{AHI} \geq 1$. The severity of OSA was graded using the international convention as follows: mild, AHI $1-4.9 / \mathrm{h}$; moderate, AHI 5-9.9/h; and severe, AHI $\geq 10 / \mathrm{h}$. Obstructive apnea was defined as decreases for at least $90 \%$ of oronasal airflow signal lasting for the duration of at least two breaths despite respiratory effort. Central apneas were defined as the lack of airflow with respiratory effort for two or more breath cycles or more than $20 \mathrm{~s}$, or shorter duration with accompanying desaturation or arousal. A value of $\geq 5$ central events per hour was accepted as clinically 
significant central apnea. Predominantly, central sleep apnea was defined as the existence of central AHI $>1$ in children with obstructive $\mathrm{AHI}<1$ [30]. Mixed apnea was defined as apnea that generally started as central apnea, followed by obstructive apnea, for at least two breath cycles. Hypopnea was defined as a reduction in nasal flow by $\geq 30 \%$ associated with an oxygen desaturation of $\geq 3 \%$ and/or an arousal awakening. The arousal index was defined as the number of arousals from sleep per hour. Mean and minimum $\mathrm{SpO}_{2}$ (oxygen saturation) values were calculated.

\section{Statistical analysis}

Statistical analysis was performed using the IBM SPSS for Windows version 23 package. The normality of numerical data was assessed using the Shapiro-Wilk test. Normally distributed continuous data are summarized using mean \pm standard deviation, and non-normally distributed continuous data are summarized as median [25-75th percentiles]. Categorical variables are shown as numbers and percentages. The chi-square test or Fisher's exact test were used to detect relations between categorical variables. The Mann-Whitney $U$ test was used to compare two independent groups in terms of numerical data. Spearman's correlation coefficient was performed to evaluate the association between numerical data. Logistic regression was performed to determine the relationship between binary variables and independent factors. A 2 -tailed $P$-value of 0.05 was considered significant.

\section{Results}

Full PSG data were present for 81 children with DS. Eight patients had excluded from the study due to TST of fewer than $4 \mathrm{~h}$ or a technically inadequate PSG. The demographic findings of the children with DS are summarized in Table 1. The mean age of the group was $4.8 \pm 3.9$ years. Fifty $(61.7 \%)$ were boys. Twenty-six (32.1\%) were aged under 2 years (range, 3 months-15.6 years). The main reasons for the first referral were as follows: routine screening in $40(49.4 \%)$, symptoms detected during routine visits in $22(27.2 \%)$, by otolaryngologist due to prolongation of upper airway symptoms in six (7.4\%), and others. The mean waiting time for the PSG appointment was 6 months. Sixty-nine $(85.1 \%)$ children had at least one comorbidity related to DS.

The baseline PSG characteristics of the patients are summarized in Table 2. Forty-three (53.1\%) had severe OSA. Eighteen $(41.9 \%)$ patients aged under 2 years had severe OSA. Clinically significant central apnea (CAI $\geq 5$ events/h) was present in $10(12.3 \%)$ children, four (40\%) of whom were older than 2 years, and four had no symptoms
Table 1 The demographic findings of children with Down syndrome referred for polysomnography

\begin{tabular}{ll}
\hline Variables & Total population $N=\mathbf{8 1}$ \\
\hline Age at study, years, mean $\pm \mathrm{SD}^{\mathrm{a}}$ & $4.8 \pm 3.9$ \\
Male, $(n, \%)$ & $50(61.7)$ \\
Body mass index z-score, mean $\pm \mathrm{SD}\left(\mathrm{kg} / \mathrm{m}^{2}\right)$ (for those aged $>2$ years) ${ }^{\mathrm{a}}$ & $-0.077 \pm 1.49$ \\
Body mass index centiles, mean $\pm \mathrm{SD}\left(\right.$ for those aged $>2$ years) ${ }^{\mathrm{a}}$ & $50.07 \pm 33.04$ \\
Nutritional status $(n, \%)$ & $10(12.3)$ \\
- Obese & $6(7.4)$ \\
- Overweight & $8(9.9)$ \\
- Underweight & \\
Comorbidity $(n, \%)$ & $20(24.7)$ \\
- Prematurity & $10(12.3)$ \\
- GERD & $10(12.3)$ \\
- Swallowing disorders & $27(33.3)$ \\
- Hypothyroidism & $39(48.1)$ \\
- Congenital heart disease & $9(11.1)$ \\
- Asthma and/or allergies & $2(2.5)$ \\
- Scoliosis & $12(14.8)$ \\
- Pectus deformity & $5(6.2)$ \\
- Pulmonary hypertension & \\
Adenotonsillar hypertrophy $(n, \%)$ & $35(43.2)$ \\
Macroglossia $(n, \%)$ & $27(33.3)$ \\
Previous adenoidectomy and/or tonsillectomy $(n, \%)$ & $8(9.9)$ \\
Second-hand smoke exposure $(n, \%)$ & $19(23.5)$ \\
\hline
\end{tabular}

Data are presented as the number (\%). Prematurity: born before 37 weeks of gestation. Pulmonary hypertension: pulmonary arterial pressures higher than $25 \mathrm{~mm} \mathrm{Hg}$ at echocardiogram

$G E R D$ gastrointestinal reflux disease

${ }^{\mathrm{a}}$ Mean \pm SD 
Table 2 Overall baseline results of children with Down syndrome referred for polysomnography

\begin{tabular}{|c|c|}
\hline Variables & Total population $N=81$ \\
\hline Age at study, years, mean $\pm S^{a}$ & $4.8 \pm 3.9$ \\
\hline $\operatorname{TST}(\min )^{\mathrm{a}}$ & $313 \pm 52.52$ \\
\hline Arousal index $(N / \mathrm{hr})^{\mathrm{a}}$ & $12 \pm 6.31$ \\
\hline $\begin{array}{l}\text { AHI } \dagger \\
\text { - AHI } \\
\text { - REM AHI } \\
\text { - Non-REM AHI }\end{array}$ & $\begin{array}{l}20.01 \pm 31.2 \\
38.98 \pm 41 \\
15.96 \pm 30.5\end{array}$ \\
\hline $\begin{array}{l}\text { OSA severity distribution }(n, \%) \\
\text { - Normal } \\
\text { - Mild } \\
\text { - Moderate } \\
\text { - Severe }\end{array}$ & $\begin{array}{l}2(2.5) \\
20(24.7) \\
16(19.8) \\
43(53.1)\end{array}$ \\
\hline Central apnea index (events/h) ${ }^{a}$ & $3.07 \pm 7.37$ \\
\hline Central apnea index $>5$ events $/ \mathrm{h} n(\%)$ & $10(12.3)$ \\
\hline REM latency $(\min )^{\mathrm{a}}$ & $156 \pm 96.54$ \\
\hline $\operatorname{REM}(\%)^{\mathrm{a}}$ & $13.84 \pm 7.01$ \\
\hline Mean $\mathrm{SpO}_{2}(\%)^{\mathrm{a}}$ & $94.23 \pm 2.6$ \\
\hline Minimal $\mathrm{SpO}_{2}(\%)^{\mathrm{a}}$ & $83.42 \pm 8.51$ \\
\hline$\%$ night with $\mathrm{SpO}_{2}<90 \%$ a & $6.05 \pm 16.31$ \\
\hline
\end{tabular}

Data are presented as the number (\%)

$A H I$ apnea-hypopnea index, TST total sleep time, REM rapid eye movement, $\mathrm{SpO}_{2}$ oxygen saturation

${ }^{\mathrm{a}}$ mean $\pm \mathrm{SD}$

associated with a sleep abnormality. Also, predominantly central sleep apnea was present in $43(53.1 \%)$ children, 25 $(58.1 \%)$ of whom were aged $>2$ years. No significant differences were found between children aged under 2 years and the others for clinically significant central apnea and predominantly central apnea $(P=0.068$ and 0.058 , respectively). The Spearman rho coefficient for the correlation between the age and central apnea index demonstrated a statistically significant association $(r=-0.34 ; P=0.002)$. Eight $(9.9 \%)$ children had upper airway surgery (adenoidectomy and/or tonsillectomy) before referral for PSG, all of whom had confirmed OSA; six had severe OSA.

Parents reported snoring in $38(46.9 \%)$ children, witnessed apnea in 26 (32.1\%), frequent night awakening in 29 (35.8\%), and sleepiness in $13(16 \%)$ children. Sleep-related symptoms (apnea and/or snoring and/or frequent night awakening) were documented in $51(63 \%)$ children. Thirty children (37\%) had a negative history of symptoms of sleep apnea, despite 12 (40\%) of them having severe OSA. Nine of the 12 children without a history of symptoms and severe OSA were aged under 4 years. Family interviews documented that $70(86 \%)$ parents had no previous information regarding possible sleep breathing disorders (SBDs) in DS until they were referred to the sleep unit. Seventeen (21\%) caregivers were not aware of sleep-related symptoms until their evaluation in the sleep unit, stating that they assumed that the mentioned symptoms might be normal for children with DS.

To predict risk factors for severe OSA, patients were categorized into two groups according to AHI: severe OSA group $(\mathrm{AHI} \geq 10)$ and others $(\mathrm{AHI}<10)$. There was no association between the presence of severe OSA and age, sex, referral reason, BMI z-score, overweight/obesity, second-hand smoke exposure, sleep-related symptoms, and comorbidities. Also, there was no significant association between the number of comorbidities and the presence of severe OSA $(P=0.096)$. In the logistic regression analysis conducted to identify the influence of predictive variables (age, sleep-related symptoms, obesity, numbers of comorbidities and second-hand smoke), there were no significant factors determined to predict severe OSA. Also, the patients were categorized according to AHI: moderate and severe OSA group $(\mathrm{AHI} \geq 5)$ and others $(\mathrm{AHI}<5)$. A significant association was demonstrated only between congenital heart diseases and AHI $\geq 5(P=0.029)$. Table 3 summarizes the possible risk factors and their association with $\mathrm{AHI}$, and Table 4 demonstrates logistic regression of demographic and clinical parameters for predictive sleep apnea.

Also, AHI was analyzed as a numerical variable. There was a negative association between the AHI score of patients aged $<2$ years and others (mean AHI in $<2$ years old group: $28.51 \pm 35.26$ and, for $\geq 2$ years old: $15.98 \pm 28.58$, $P=0.023)$. Moreover, there was a significant association between the obese/overweight group and others (mean AHI in obese/overweight: $21.54 \pm 17.34$ and others: $19.6 \pm 33.87$, $P=0.018)$. There was a significant relationship between congenital heart diseases $(P=0.013)$ and the number of comorbidities $(r=0.25 ; P=0.019)$ and AHI. There was no significant association between the AHI score and the other parameters. Only patients with pulmonary hypertension $(P=0.057)$ and GERD $(P=0.055)$ had a smaller $P$-value, but it was not significant.

Patients were analyzed in two groups according to the presence of sleep-related symptoms (apnea and/or snoring and/or frequent night awakening). As presented in Table 5, there were no significant differences between symptomatic and asymptomatic patients in terms of sex, comorbidities, and baseline PSG findings. However, patients who were exposed to second-hand smoke had significantly more sleeprelated symptoms than the others $(P=0.001)$. Moreover, sleep-related symptoms were significantly more frequent in children who were older and who experienced frequent upper respiratory tract infections $(P=0.041$ and $P=0.009$, respectively).

Treatments included supportive medication, respiratory support devices, oxygen, and surgery. A continuous positive airway pressure (CPAP) titration study was offered to 
Table 3 Demographic and clinical parameters to predictive moderate and severe obstructive sleep apnea according to AHI

\begin{tabular}{|c|c|c|c|c|c|c|}
\hline Variables & $\begin{array}{l}\text { AHI }<5 \\
n=22\end{array}$ & $\begin{array}{l}\mathrm{AHI} \geq 5 \\
n=59\end{array}$ & $P$-value & $\begin{array}{l}\mathrm{AHI}<10 \\
n=38\end{array}$ & $\begin{array}{l}\mathrm{AHI} \geq 10 \\
n=43\end{array}$ & $P$-value \\
\hline Age at study, years, mean \pm SD & $5.49 \pm 3.57$ & $4.6 \pm 4.12$ & 0.14 & $4.94 \pm 3.46$ & $4.75 \pm 4.45$ & 0.32 \\
\hline Male $^{\mathrm{a}}$ & $17(77.27)$ & $33(55.93)$ & 0.13 & $24(63.15)$ & $26(60.46)$ & 0.098 \\
\hline Body mass index $\mathrm{z}$-score, mean $\pm \mathrm{SD}(>2$ years $)$ & $-0.22 \pm 1.2$ & $0 \pm 16$ & 0.43 & $-0.03 \pm 1.5$ & $-0.13 \pm 1.51$ & 0.72 \\
\hline Body mass index centile, mean $\pm \mathrm{SD}$ ( $>2$ years $)$ & $43.78 \pm 29.47$ & $53.38 \pm 34.71$ & 0.3 & $51.16 \pm 31.05$ & $48.76 \pm 35.89$ & 0.78 \\
\hline Obese/overweight $\mathrm{t}^{\mathrm{a}}$ & $1(4.54)$ & $15(25.42)$ & 0.057 & $4(10.5)$ & $12(27.9)$ & 0.093 \\
\hline \multicolumn{7}{|l|}{ Comorbidity $^{\mathrm{a}}$} \\
\hline - Prematurity & $4(18.18)$ & $16(27.11)$ & 0.53 & $10(26.31)$ & $10(23.25)$ & 0.94 \\
\hline • GERD & $1(4.54)$ & $9(15.25)$ & 0.27 & $3(7,89)$ & $7(16.27)$ & 0.32 \\
\hline - Swallowing disorders & $2(9.09)$ & $8(13.55)$ & 0.72 & $4(10,52)$ & $6(13.95)$ & 0.74 \\
\hline • Hypothyroidism & $5(22.7)$ & $22(37.28)$ & 0.3 & $10(26.31)$ & $17(39.53)$ & 0.27 \\
\hline - Congenital heart disease & $6(27.27)$ & $33(55.93)$ & 0.029 & $15(39.47)$ & $24(55.81)$ & 0.41 \\
\hline - Pectus deformity & $3(13.63)$ & $9(15.25)$ & 0.999 & $6(15.78)$ & $6(13.95)$ & 0.999 \\
\hline - Pulmonary hypertension & $1(1.54)$ & 46.77) & 0.999 & $1(2.63)$ & $4(9.3)$ & 0.36 \\
\hline \multicolumn{7}{|l|}{ Symptoms $^{\mathrm{a}}$} \\
\hline - Snoring & $9(40.9)$ & $29(49.15)$ & 0.68 & $16(42.1)$ & $22(51.16)$ & 0.55 \\
\hline - Apnea & $5(22.72)$ & $21(35.59)$ & 0.37 & $9(23.68)$ & $17(39.53)$ & 0.14 \\
\hline - Frequent night awakening & $6(27.27)$ & $23(38.98)$ & 0.47 & $11(28.94)$ & $18(41.86)$ & 0.32 \\
\hline - Day time sleepiness & $2(9.09)$ & $11(18.64)$ & 0.49 & $4(10.52)$ & $9(20.93)$ & 0.999 \\
\hline - Sleep abnormality-related symptoms & $12(54.54)$ & $39(66.1)$ & 0.48 & $22(57.89)$ & $29(67.44)$ & 0.51 \\
\hline Adenotonsillar hypertrophy ${ }^{\mathrm{a}}$ & $8(36.36)$ & $27(45.76)$ & 0.4 & $15(39.47)$ & $20(46.51)$ & 0.54 \\
\hline Second-hand smoking ${ }^{\mathrm{a}}$ & $3(13.63)$ & $16(27.11)$ & 0.3 & $8(21.05)$ & $11(25.58)$ & 0.85 \\
\hline
\end{tabular}

Sleep abnormality-related symptoms; apnea and/or snoring and/or frequent night awakening GERD gastrointestinal reflux disease, $P S G$ polysomnography, $\mathrm{SpO}_{2}$ oxygen saturation, $S D$ standard deviation ${ }^{\mathrm{a}}$ Data are presented as the number $(\%)$ and mean $\pm \mathrm{SD}$

51 children after supportive medication and/or otolaryngologic evaluation. Adenotonsillectomy was recommended to nine patients; however, it was performed for five children after the PSG. Of the 31 patients who were recommended to have CPAP/BIPAP, 22 families acquired the device.

\section{Discussion}

The number of patients referred to sleep units has risen recently; however, the global need is not sufficiently met. As the results of our study revealed, patients had to wait
Table 4 Logistic regression of demographic and clinical parameters for predictive moderate and severe sleep apnea

\begin{tabular}{|c|c|c|c|c|c|c|c|c|}
\hline \multirow[t]{2}{*}{ Variables } & \multicolumn{4}{|l|}{$\mathbf{A H I} \geq 5$} & \multicolumn{4}{|c|}{$\mathbf{A H I} \geq 10$} \\
\hline & $\beta$ & OR & $P$-value & $95 \% \mathrm{CI}$ & $\beta$ & OR & $P$-value & $95 \% \mathrm{CI}$ \\
\hline Sex & -1.02 & 0.35 & 0.15 & $0.087-1.47$ & 0.15 & 1.16 & 0.77 & $0.39-3.47$ \\
\hline Age group & 1.64 & 5.19 & 0.047 & $1.02-26.35$ & 0.93 & 2.53 & 0.15 & $0.71-9.06$ \\
\hline Obese/overweight & 2.14 & 8.55 & 0.062 & $0.89-81.62$ & 0.87 & 2.39 & 0.2 & $0.62-9.1$ \\
\hline Number of comorbidities & -0.061 & 1.11 & 0.68 & $0.66-1.64$ & 0.054 & 1.05 & 0.8 & $0.61-4.88$ \\
\hline Sleep-related symptom & 0.78 & 2.18 & 0.28 & $0.52-9.19$ & 0.37 & 1.45 & 0.54 & $0.43-4.87$ \\
\hline Second-hand smoking & 0.76 & 2.15 & 0.38 & $0.38-12$ & 0.28 & 1.32 & 0.68 & $0.33-5.2$ \\
\hline Adenotonsillar hypertrophy & 0.38 & 1.47 & 0.56 & $0.39-5.43$ & 0.54 & 1.72 & 0.3 & $0.61-4.88$ \\
\hline
\end{tabular}

Age group: patients analyzed in two groups according two age; $<2$ years old and $\geq 2$ years old, sleeprelated symptoms: apnea and/or snoring and/or frequent night awakening, comorbidities (congenital heart disease (CHD), hypothyroidism, feeding problems, anatomic functional and gastrointestinal (GI) conditions, asthma and /or allergic rhinitis, respiratory and airway problems)

$O R$ odds ratio 
Table 5 Demographic, clinical and polysomnographic parameters for children with and without sleep-related symptoms

\begin{tabular}{|c|c|c|c|}
\hline Variables & $\begin{array}{l}\text { Sleep-related symptom- } \\
\text { positive } \\
n=51\end{array}$ & $\begin{array}{l}\text { Sleep-related symptom- } \\
\text { negative } \\
n=30\end{array}$ & $P$-value \\
\hline Age at study, years, mean \pm SD & $5.47 \pm 4.1$ & $3.76 \pm 3.55$ & 0.041 \\
\hline Male $^{\mathrm{a}}$ & $30(58.82)$ & $20(66.66)$ & 0.64 \\
\hline $\begin{array}{l}\text { Body mass index centile, mean } \pm S D \\
\text { (for older than two years old) }\end{array}$ & $54.82 \pm 33.08$ & $38.5 \pm 30.9$ & 0.1 \\
\hline $\begin{array}{l}\text { Body mass index } \mathrm{z} \text {-score, mean } \pm \mathrm{SD} \\
\text { (for older than two years old) }\end{array}$ & $0.13 \pm 1.41$ & $-0.59 \pm 1.6$ & 0.093 \\
\hline Obese/overweight $^{\mathrm{a}}$ & $11(21.6)$ & $5(16.6)$ & 0.80 \\
\hline \multicolumn{4}{|l|}{ Comorbidity $^{\mathrm{a}}$} \\
\hline - Prematurity & $16(31.37)$ & $4(13.33)$ & 0.12 \\
\hline - GERD & $6(11.76)$ & $4(13.33)$ & 0.999 \\
\hline - Swallowing disorders & $7(13.72)$ & $3(10)$ & 0.73 \\
\hline • Hypothyroidism & $18(35.29)$ & $9(30)$ & 0.76 \\
\hline - Congenital heart disease & $27(52.94)$ & $12(40)$ & 0.53 \\
\hline - Pectus deformity & $8(16.68)$ & $4(13.33)$ & 0.999 \\
\hline - Pulmonary hypertension & $5(9.8)$ & 0 & 0.15 \\
\hline Adenotonsillar hypertrophy ${ }^{\mathrm{a}}$ & $27(52.94)$ & $8(26.66)$ & 0.13 \\
\hline Second-hand smoking ${ }^{\mathrm{a}}$ & $18(35.29)$ & $1(3.33)$ & 0.001 \\
\hline Frequent upper respiratory infection ${ }^{\mathrm{a}}$ & $15(29.41)$ & $1(3.33)$ & 0.009 \\
\hline \multicolumn{4}{|l|}{ PSG findings } \\
\hline Arousal index (N/h) & $11.87 \pm 6.23$ & $12.26 \pm 6.54$ & 0.83 \\
\hline AHI & $19.63 \pm 32.01$ & $20.64 \pm 30.35$ & 0.59 \\
\hline Severe OSA $(A H I \geq 10)^{\mathrm{a}}$ & $29(56.86)$ & $14(46.66)$ & 0.51 \\
\hline Central apnea index $>5$ events $/ \mathrm{h}^{\mathrm{a}}$ & $6(11.76)$ & $4(13.33)$ & 0.999 \\
\hline Predominantly central sleep apnea ${ }^{a}$ & $29(56.86)$ & $14(46.66)$ & 0.51 \\
\hline REM (\%) & $13.46 \pm 6.45$ & $14.48 \pm 7.95$ & 0.34 \\
\hline Mean $\mathrm{SpO}_{2}(\%)$ & $94.25 \pm 2.71$ & $94.2 \pm 2.53$ & 0.95 \\
\hline Minimal $\mathrm{SpO}_{2}(\%)$ & $83.01 \pm 9.32$ & $84.1 \pm 7.05$ & 0.57 \\
\hline$\%$ night with $\mathrm{SpO}_{2}<90 \%$ & $5.97 \pm 15.30$ & $6.19 \pm 18.15$ & 0.4 \\
\hline
\end{tabular}

Sleep-related symptoms: apnea and/or snoring and/or frequent night awakening

GERD gastrointestinal reflux Disease, OSA obstructive sleep apnea, AHI apnea-hypopnea index, REM rapid eye movement, $\mathrm{SpO}_{2}$ oxygen saturation, $\mathrm{SD}$ standard deviation

${ }^{a}$ Data are presented as the number (\%), and mean \pm SD for PSG, which renders the prediction of patients with severe OSA quite significant. Accordingly, studies conducted to this end have been limited in number, and their conclusions have been contradictory. The results of our study revealed, contrary to our initial hypothesis, that we were not able to predict severe OSA in children with DS through age, sex, exposure to second-hand smoke, clinical findings, anthropometric features, and the presence of comorbidities.

Although the correlation between obesity and the significance of sleep apnea in adult patients with DS has been proven, such a correlation in children with DS is not clear. Although some studies have not been able to demonstrate a relationship between BMI and SBD [19, 31, 32], others, such as that by Basil et al., found that moderate and severe OSA was more frequent in patients with obesity [27]. In a study by Chamseddin et al., children with obesity were found to have severe OSA more frequently [25]. Despite that the AHI scores of the obese/overweight group were higher than the others, interestingly, no significant relationship between BMI percentiles, BMI-z-scores, and overweight/obesity, and moderate or severe OSA was detected in the present study.

There may be various reasons why such a relationship is seen among adults, but it varies among children; it is important to clarify this point through further studies. We believe that this situation stems from the facts that OSA is not affected only by obesity and DS has multiple predisposing factors for OSA, apart from obesity. As a result, determining the contribution of obesity in DS requires studies with larger groups of participants.

As is the case with obesity, the relationship between predisposing comorbid conditions and OSA in children with DS 
remains obscure in limited studies. In their study conducted with infants with DS, Goffinski et al. argued that there was a relationship between OSA and certain comorbid conditions such as CHD, prematurity, and GERD. However, very few patients in the study received full-night PSG [23]. On the other hand, Diez et al. found no relationship between CHD and SBD in their study [31]. Even though comorbidity presence was questioned on a large scale in the present study, we failed to identify a relationship between comorbid conditions and severe OSA. It was notable that the increasing number of comorbid conditions was not associated with severe OSA. There was only a relationship between congenital heart disease and the number of comorbidities detected for $\mathrm{AHI} \geq 5$. However, there was no significant relation in regression analysis. The relation between age group and AHI $\geq 5$ was explained by the fact that young children with severe OSA findings were referred for PSG.

Diez et al. found that male sex, being younger than 8 years, and tonsillar hypertrophy predicted OSA [31], and Dyken et al. reported that older age and elevated BMI could be associated with more severe OSA in their study conducted with 19 patients [10]. Yet, in a study conducted to ascertain predictors for OSA by Hills et al., the authors found no correlations between OSA and age, BMI, and tonsil size [33]. Only a few of the studies in the literature specifically aimed to predict severe OSA, as was the case in our study. It was, however, quite challenging to predict OSA, especially severe OSA in patients with DS, as was shown by the examples and the results of our study. There may be many reasons why the prediction of severe OSA is challenging because DS manifests many of the predisposing conditions to OSA. However, our results indicate that congenital heart diseases and the number of comorbidities may be important in predicting moderate to severe OSA, and more studies are needed on this subject.

To the best of our knowledge, our study is the first to show the relationship between smoke exposure and an increase in sleep-related symptoms. Our results revealed no significant differences in other parameters such as sex, anthropometric measures, comorbidities, and PSG features between symptomatic and asymptomatic patients. Similarly, Howard et al. reported no association between reported symptoms and the degree of AHI [11]. We found that $37 \%$ of children with OSA had no symptoms related to sleep abnormalities. Also, high rates of severe OSA were prevalent, even in children without a history of SBD (40\%), in line with the results of Maris et al. (53.8\%) [34]. The fact that severe OSA was seen in patients with DS, including the asymptomatic patients, reveals the importance of PSG screening in such patients.

The results of our study and others indicate that OSA is common in children with DS, and more than half (53.1\%) have severe disease, as was the case in the study conducted by Maris et al. (52\%) (34). Studies in the literature reported varying results regarding the prevalence of OSA (33). There may be many reasons for such differences; the characteristics of the patient population, the type of PSG performed, the definition of sleep apnea, and the methodological differences in the studies. Our study included patients with a wide age range, the majority of whom had been referred because of sleep-related symptoms, and the sleep unit in the study was based at a large referral center. Moreover, full-night PSG was performed on all children. The prevalence of OSA might have been found higher than those of some other studies in the literature due to these reasons.

To the best of our knowledge, our study is the first to show that the caregivers of patients with DS had very little knowledge of sleep disorders. A great majority of parents (89\%) did not know about sleep disorders until they were referred to the sleep unit. Previous studies demonstrated the underestimation of sleep-related symptoms by parents of children with DS [19, 35]. Some of the parents interviewed within the scope of our study indicated that they were not aware of their children experiencing sleep-related symptoms or believed that such symptoms were normal for their children. Such underestimation might be based on their lack of information. Offering information to parents at the onset of diagnosis can lead to better observations of OSA symptoms. It is, therefore, important for physicians performing routine follow-ups of children with DS to be adequately sensitive about this matter.

The prevalence of CSA ranges between 1 and 5\% in the pediatric age group; it is seen rarely after the age of 2 years due to maturation of the central nervous system [36]. The association of CSA and certain diseases such as the PraderWilli syndrome, Arnold-Chiari malformations, and achondroplasia was reported previously [36, 37]. However, only a few reported increases in the frequency of CSA in DS [30, 38]. Our results demonstrated that CSA in children with DS was twice as common than in other children. Moreover, we also documented a high frequency of predominant central apneas. However, even though the central apnea index is higher at younger ages, that there was no significant difference in patients younger and older than 2 years proves to be one of the interesting results of our study. In a study by Fan et al., the central apnea ratio was higher in children younger than 3 years of age. The authors argued that central apneas could be related to immature respiratory central control and hypotonia seen in early childhood based on this finding [39]. In another study, in which 10 patients with DS were compared with patients with fragile $\mathrm{X}$ syndrome, it was reported that central apneas were predominant in patients with DS and that it increased by age. The authors suggested that the underlying cause of this might be brainstem dysfunction [38]. Similarly, the results of our study emphasize the importance of central apneas, but the relationship between 
central apnea and age in DS still needs to be clarified by a larger cohort in prospective studies.

In our study, it was also observed that adenotonsillectomy procedures were not often performed, despite about half of our patients having severe OSA. Some studies reported that upper respiratory system procedures had limited benefits for the treatment of OSA in patients with DS [40]. Hill et al. stated that physicians might be reserved about the issue due to the limited information about OSA-related morbidities in children with DS, along with the limited number of studies on DS and tonsillectomy [33]. Further prospective studies are needed to clarify this issue.

Our study had several limitations. First, the study population age group represented a wide range. We included all children with DS regardless of the reason for referral, which might have caused bias. Also, our study was conducted at a large referral hospital in Turkey; therefore, the disease severity could have been different from the general population of children with DS. Also, children with and without upper airway surgery were included. We did not include overnight carbon dioxide measurements because they were absent in more than half of the patients; thus, we could not evaluate hypoventilation. A standardized sleep questionnaire could not be performed because of the long duration of the study period and the COVID-19 pandemic, which is one of the most significant limitations of our investigation. Although demographic and PSG data were retrospectively collected in our study, all retrospective data were confirmed through phone interviews with the families. The strength of our study was the questioning of important points such as the families' levels of information, retrospective awareness by phone interview.

\section{Conclusion}

Our results demonstrated a high prevalence of severe OSA in children with DS, even in patients without a history of SBD, and also in young children. Severe OSA could not be predicted in children with DS based on demographic characteristics, symptoms, and comorbid conditions, underlining the importance of routine screening with PSG for such patients beginning at very young ages. Obstructive apneas and central and predominantly central apneas could be a part of the spectrum of sleep abnormalities in DS. Furthermore, the present study illustrates the importance of offering information to parents about SBD during routine follow-ups.

Authors' contributions Mina Hizal: conceptualization, methodology, writing-original draft; Ozlem satırer: investigation; Sanem Eryilmaz Polat: investigation; Dilber Ademhan Tural: investigation; Beste Ozsezen: investigation; Birce Sunman: investigation; Sevilay Karahan: formal analysis, data curation; Nagehan Emiralioglu: visualization, review and editing; Pelin Ozlem Simsek Kiper: writing — review and editing; Gulen Eda Utine: writing — review and editing; Koray Boduroglu: writing—review and editing; Ebru Yalcin: validation, writing — review and editing; Deniz Dogru: writingreview and editing; Nural Kiper: writing—review and editing; Ugur Ozcelik: conceptualization, supervision.

\section{Availability of data and material Yes.}

Code availability N/A

\section{Declarations}

Ethics approval Yes.

Consent to participate Yes.

Consent for publication N/A

Competing interests The authors have no competing interests.

\section{References}

1. Parker SE, Mai CT, Canfield MA, Rickard R, Wang Y, Meyer RE et al (2010) Updated National Birth Prevalence estimates for selected birth defects in the United States, 2004-2006. Birth Defects Res A Clin Mol Teratol 88(12):1008-1016

2. de Graaf G, Buckley F, Skotko BG (2015) Estimates of the live births, natural losses, and elective terminations with Down syndrome in the United States. Am J Med Genet A 167A(4):756-767

3. Dumortier L, Bricout VA (2020) Obstructive sleep apnea syndrome in adults with down syndrome: Causes and consequences. Is it a "chicken and egg" question? Neurosci Biobehav Rev 108:124-38

4. Konstantinopoulou S, Tapia IE, Kim JY, Xanthopoulos MS, Radcliffe J, Cohen MS et al (2016) Relationship between obstructive sleep apnea cardiac complications and sleepiness in children with Down syndrome. Sleep Med 17:18-24

5. Weijerman ME, de Winter JP (2010) Clinical practice. The care of children with Down syndrome. Eur J Pediatr 169(12):1445-52

6. Waters KA, Castro C, Chawla J (2020) The spectrum of obstructive sleep apnea in infants and children with Down Syndrome. Int J Pediatr Otorhinolaryngol 129:109763

7. Ikizoglu NB, Kiyan E, Polat B, Ay P, Karadag B, Ersu R (2019) Are home sleep studies useful in diagnosing obstructive sleep apnea in children with down syndrome? Pediatr Pulmonol 54(10):1541-1546

8. Uong EC, McDonough JM, Tayag-Kier CE, Zhao H, Haselgrove J, Mahboubi S et al (2001) Magnetic resonance imaging of the upper airway in children with Down syndrome. Am J Respir Crit Care Med 163(3 Pt 1):731-736

9. Guimaraes CV, Donnelly LF, Shott SR, Amin RS, Kalra M (2008) Relative rather than absolute macroglossia in patients with Down syndrome: implications for treatment of obstructive sleep apnea. Pediatr Radiol 38(10):1062-1067

10. Dyken ME, Lin-Dyken DC, Poulton S, Zimmerman MB, Sedars E (2003) Prospective polysomnographic analysis of obstructive sleep apnea in down syndrome. Arch Pediatr Adolesc Med 157(7):655-660

11. Howard JJM, Sarber KM, Yu W, Smith DF, Tikhtman RO, Simakajornboon N et al (2020) Outcomes in children with down syndrome and mild obstructive sleep apnea treated non-surgically. Laryngoscope 130(7):1828-1835 
12. Trois MS, Capone GT, Lutz JA, Melendres MC, Schwartz AR, Collop NA et al (2009) Obstructive sleep apnea in adults with Down syndrome. J Clin Sleep Med 5(4):317-323

13. Churchill SS, Kieckhefer GM, Landis CA, Ward TM (2012) Sleep measurement and monitoring in children with Down syndrome: a review of the literature, 1960-2010. Sleep Med Rev 16(5):477-488

14. Bruni O, Ferri R (2009) Neurocognitive deficits in pediatric obstructive sleep apnea: a multifaceted pathogenetic model. Sleep Med 10(2):161-163

15. Beebe DW (2006) Neurobehavioral morbidity associated with disordered breathing during sleep in children: a comprehensive review. Sleep 29(9):1115-1134

16. Brockmann PE, Bertrand P, Pardo T, Cerda J, Reyes B, Holmgren NL (2012) Prevalence of habitual snoring and associated neurocognitive consequences among Chilean school aged children. Int J Pediatr Otorhinolaryngol 76(9):1327-1331

17. Breslin J, Spano G, Bootzin R, Anand P, Nadel L, Edgin J (2014) Obstructive sleep apnea syndrome and cognition in Down syndrome. Dev Med Child Neurol 56(7):657-664

18. Bull MJ (2011) Committee on G. Health supervision for children with Down syndrome. Pediatrics 128(2):393-406

19. Shott SR, Amin R, Chini B, Heubi C, Hotze S, Akers R (2006) Obstructive sleep apnea: Should all children with Down syndrome be tested? Arch Otolaryngol Head Neck Surg 132(4):432-436

20. Skotko BG, Macklin EA, Muselli M, Voelz L, McDonough ME, Davidson E et al (2017) A predictive model for obstructive sleep apnea and Down syndrome. Am J Med Genet A 173(4):889-896

21. Brockmann PE, Damiani F, Nunez F, Moya A, Pincheira E, Paul MA et al (2016) Sleep-disordered breathing in children with Down syndrome: usefulness of home polysomnography. Int J Pediatr Otorhinolaryngol 83:47-50

22. Brockmann PE, Perez JL, Moya A (2013) Feasibility of unattended home polysomnography in children with sleep-disordered breathing. Int J Pediatr Otorhinolaryngol 77(12):1960-1964

23. Goffinski A, Stanley MA, Shepherd N, Duvall N, Jenkinson SB, Davis $C$ et al (2015) Obstructive sleep apnea in young infants with Down syndrome evaluated in a Down syndrome specialty clinic. Am J Med Genet A 167A(2):324-330

24. Lal C, White DR, Joseph JE, van Bakergem K, LaRosa A (2015) Sleep-disordered breathing in Down syndrome. Chest 147(2):570-579

25. Chamseddin BH, Johnson RF, Mitchell RB (2019) Obstructive sleep apnea in children with Down Syndrome: demographic, clinical, and polysomnographic features. Otolaryngol Head Neck Surg 160(1):150-157

26. Zemel BS, Pipan M, Stallings VA, Hall W, Schadt K, Freedman DS et al (2015) Growth charts for children with Down syndrome in the United States. Pediatrics 136(5):e1204-e1211
27. Basil JS, Santoro SL, Martin LJ, Healy KW, Chini BA, Saal HM (2016) Retrospective study of obesity in children with Down syndrome. J Pediatr 173:143-148

28. Brodsky L (1989) Modern assessment of tonsils and adenoids. Pediatr Clin North Am 36(6):1551-1569

29. Berry RB, Budhiraja R, Gottlieb DJ, Gozal D, Iber C, Kapur VK et al (2012) Rules for scoring respiratory events in sleep: update of the 2007 AASM Manual for the Scoring of Sleep and Associated Events. Deliberations of the Sleep Apnea Definitions Task Force of the American Academy of Sleep Medicine. J Clin Sleep Med 8(5):597-619

30. Trucco F, Chatwin M, Semple T, Rosenthal M, Bush A, Tan HL (2018) Sleep disordered breathing and ventilatory support in children with Down syndrome. Pediatr Pulmonol 53(10):1414-1421

31. de Miguel-Diez J, Villa-Asensi JR, Alvarez-Sala JL (2003) Prevalence of sleep-disordered breathing in children with Down syndrome: polygraphic findings in 108 children. Sleep 26(8):1006-1009

32. Fitzgerald DA, Paul A, Richmond C (2007) Severity of obstructive apnoea in children with Down syndrome who snore. Arch Dis Child 92(5):423-425

33. Hill CM, Evans HJ, Elphick H, Farquhar M, Pickering RM, Kingshott R et al (2016) Prevalence and predictors of obstructive sleep apnoea in young children with Down syndrome. Sleep Med 27-28:99-106

34. Maris M, Verhulst S, Wojciechowski M, Van de Heyning P, Boudewyns A (2016) Prevalence of obstructive sleep apnea in children with Down syndrome. Sleep 39(3):699-704

35. Marcus CL, Keens TG, Bautista DB, von Pechmann WS, Ward SL (1991) Obstructive sleep apnea in children with Down syndrome. Pediatrics 88(1):132-139

36. McLaren AT, Bin-Hasan S, Narang I (2019) Diagnosis, management and pathophysiology of central sleep apnea in children. Paediatr Respir Rev 30:49-57

37. Patel DM, Rocque BG, Hopson B, Arynchyna A, Bishop ER, Lozano D et al (2015) Sleep-disordered breathing in patients with myelomeningocele. J Neurosurg Pediatr 16(1):30-35

38. Ferri R, Curzi-Dascalova L, Del Gracco S, Elia M, Musumeci SA, Stefanini MC (1997) Respiratory patterns during sleep in Down's syndrome:importance of central apnoeas. J Sleep Res 6(2):134-141

39. Fan Z, Ahn M, Roth HL, Li L, Vaughn BV (2017) Sleep apnea and hypoventilation in patients with Down syndrome: analysis of 144 polysomnogram studies. Children (Basel) 4(7)

40. Dudoignon B, Amaddeo A, Frapin A, Thierry B, de Sanctis L, Arroyo JO et al (2017) Obstructive sleep apnea in Down syndrome: benefits of surgery and noninvasive respiratory support. Am J Med Genet A 173(8):2074-2080

Publisher's Note Springer Nature remains neutral with regard to jurisdictional claims in published maps and institutional affiliations. 